\title{
Probability distribution functions for solar and stellar magnetic fields
}

\author{
Jan O. Stenflo \\ Institute of Astronomy, ETH Zurich, CH-8093 Zurich, and \\ Istituto Ricerche Solari Locarno (IRSOL), Via Patocchi, CH-6605 Locarno-Monti \\ email: stenflo@astro.phys.ethz.ch
}

\begin{abstract}
Magneto-convection structures the magnetic field in solar and stellar atmospheres over scales that for the Sun span about 8 orders of magnitudes, down to the magnetic diffusion scale of order $10 \mathrm{~m}$. The statistical properties of this structuring are governed by probability density functions (PDFs), for the vertical and transverse field components as well as for the field inclination. Due to the fractal nature of the field pattern these PDFs appear to have a high degree of scale invariance. There are serious pitfalls in the derivations of empirical PDFs, pitfalls that are particularly severe in the case of the field inclination. This explains the fragmentary and rather unreliable PDF information available in the published literature. Here we discuss the nature of these pitfalls and indicate how they may be avoided, using Hinode quiet-sun Stokes vector data to derive PDFs for the field strength and field inclination.
\end{abstract}

Keywords. Sun: magnetic fields, Sun: photosphere, techniques: polarimetric, line: formation

\section{Resolved and unresolved scales}

As we zoom in on ever smaller scales in solar magnetograms (cf. Fig. 1), we find a fractal-like pattern with a high degree of self-similarity (Stenflo \& Holzreuter 2002, 2003). It is in fact hard to guess the spatial scale of a magnetogram without tick marks, due to this scale invariance.

As ever more powerful solar telescopes come on line, we will be able to resolve smaller and smaller magnetic features, but due to the self-similarity, the appearance of the magnetic pattern would be expected to remain pretty much the same, except that the new tick marks will represent smaller scales.

As we go down in scale, the magnetic Reynolds number decreases until it reaches a value of unity around the $10 \mathrm{~m}$ scale (de Wijn et al. 2009). Below a value of unity the field ceases to be frozen in, because the time scale of magnetic diffusion becomes smaller than the dynamic time scale. As the viscous diffusion limit for the turbulent spectrum lies at even much smaller scales, we expect that magneto-convection will structure the field all the way down to the $10 \mathrm{~m}$ scale. This is 4 orders of magnitude smaller than the angular resolution limit of the best current telescopes.

On the quiet Sun we will therefore remain very far from resolving any of the magnetic structures in the foreseeable future. Analysis of Hinode data in different ways (cf. Stenflo 2009) reveals subpixel magnetic structuring far beyond the Hinode 0.3 arcsec angular resolution (see more about this below). For observed magnetic fields, which always refer to a certain finite angular resolution, we should therefore always avoid using the term "field strength" and instead use the term "flux density". Although both these quantities have the same dimension $(\mathrm{G})$, confusion can be avoided if the term "field strength" is only used for resolved fields. In the asymptotic limit of infinite resolution the two concepts "field strength" and "flux density" become identical. 

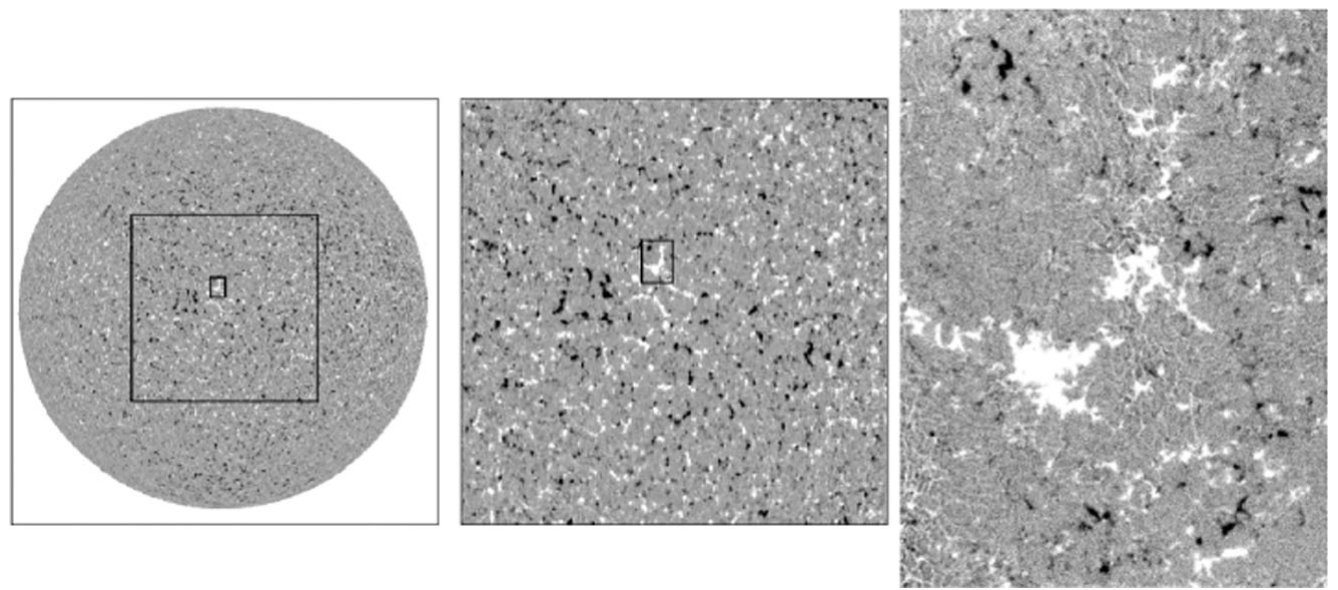

Figure 1. Fractal-like magnetic fields of the quiet Sun, observed on 9 February 1996. The two maps to the left are from a Kitt Peak full-disk magnetogram, while the right, high-resolution magnetogram was recorded the same day at the Swedish La Palma Observatory (courtesy Göran Scharmer). It covers an area that is only $0.35 \%$ of the map next to it.

\section{The concept of "intrinsic field strength"}

As it was realized already in the 1960s that the true magnetic structures were far smaller than the available resolution, indirect methods were developed to diagnose the nature of the magnetic field beyond the spatial resolution limit. The Stokes $V$ line ratio technique makes use of the differential non-linearity in the relation between field strength and circular polarization measured simultaneously in different spectral lines (Stenflo 1973). With a very special choice of line pair it is possible to isolate the magnetic effects from the thermodynamic effects. This led to the discovery that more than $90 \%$ of the magnetic flux seen with moderate angular resolution is due to subresolution kG fields. Since however the measured flux densities on the quiet Sun are only a few G, the high field strengths imply that the corresponding magnetic elements occupy on average only about $1 \%$ of the quiet-Sun photospheric volume. The theoretical counterpart of these seemingly intermittent strong-field structures became the magnetic flux tubes, which usually were assumed to be embedded in a non-magnetic environment. Ever more sophisticated flux tube models were then developed.

To determine the $\mathrm{kG}$ field strength of the spatially unresolved field structures an interpretational model based on a 2-component concept was found to be effective and to give consistent results. The concept is to assume that we have one magnetic component with a certain "intrinsic" field strength and filling factor, and one "non-magnetic" component. This 2-component model became the standard interpretational tool that was applied universally not only in solar but also in stellar physics.

With the success of this approach it tended to be forgotten that it represents an extreme idealization that filters out a certain property of the underlying reality, and that the true nature of this reality may be very different. If we would describe the 2component model in terms of probability density functions (PDFs) that are frequently used to characterize the statistical properties of the fields found in numerical simulations of magneto-convection, the 2-component PDF would appear very strange indeed: it would consist of two $\delta$ function peaks, one near a field strength of $1 \mathrm{kG}$, representing the magnetic component, while the second peak, located at zero field strength, would be about 100 times taller (since the magnetic filling factor is only of order $1 \%$ ). The huge 
and the tiny $\delta$ function peaks would be separated by a vast, empty "desert" in between. In contrast, all numerical simulations of magneto-convection produce continuous PDFs, smoothly connecting the weakest and the strongest fields.

We therefore realize that the 2-component model is a crude interpretational filter that projects out some global property of the strong-field tail of the actual PDF in terms of a single number that we call the "intrinsic field strength". What this number physically really means depends on the nature of the underlying PDF and on the functional relation between field properties and polarization. One should be careful in assigning too much physical meaning to this "intrinsic field strength" as representing the field strength of a discrete flux tube. The underlying fractal reality is likely to be different.

After the $\mathrm{kG}$ values for the magnetic component of the quiet Sun had been discovered, the attention turned towards exploring the magnetic nature of the so-called "nonmagnetic" component. It was from the start clear that it could not be truly non-magnetic, since a major field-free region in the highly conducting turbulent solar plasma is a physical impossibility. If the field is weak, the frozen-in field lines must be tangled up and amplified by the turbulent motions. The question was how strong this tangled field is.

This question could not be answered with the Zeeman effect (due to cancellation of the mixed-polarity signatures), but the introduction of the Hanle effect opened a window to this aspect of solar magnetism (Stenflo 1982), since the Hanle effect with its different symmetry properties is not subject to the mentioned cancellations. Interpreting the observed Hanle signatures in terms of a single-valued field strength, values of 30-60 G were found (Faurobert-Scholl 1993; Trujillo Bueno, Shchukina \& Asensio Ramos 2004).

Even when the 2-component model is extended by including the Hanle effect, it would, if interpreted literally in terms of a PDF, mean that the huge $\delta$ function peak at zero field strength is shifted to a location somewhere in the interval 30-60 G (while the tiny $1 \mathrm{kG}$ peak for the "magnetic component" remains). As we however have all reasons to expect that the actual PDF is a smooth function connecting in a continuous fashion the weakest with the strongest $(\mathrm{kG})$ fields, the single numbers extracted from the observed Hanle signatures must represent some filtered global property of the weak-field portion of the underlying PDF. What this single number really means depends on the nature of the PDF, the angular distribution of field vectors, and the non-linear functional relation between the Hanle polarization signature and the field properties (strength and orientation).

It is therefore clear that there is now a big need to transcend the 2-component approach and move in the direction of interpretational models based on PDFs.

\section{Angular distribution of field vectors}

We have seen that the "intrinsic properties" of PDFs cannot be determined (in any direct way at least) in the spatially unresolved domain, since the unavoidably very idealized interpretational model used gives us a filtered version of the underlying reality characterized by a couple of discrete parameters. While such parameters can provide important constraints on the nature of the underlying PDF, a full reconstruction of the PDF would heavily rely on guessed-at model assumptions. As an alternative we suggest to approach this problem by instead exploring the PDFs in the spatially resolved domain and the scaling laws that they obey.

It might seem that it would in the spatially resolved domain be a straightforward matter to extract the PDFs for the flux densities as averaged over the spatial resolution element. This averaging is however highly non-linear and therefore far from trivial, as we will illustrate for the case of the angular distribution of field vectors. Lites et al. (2008) 
analysed Hinode SOT/SP Stokes vector spectra of the Fe I 6301 and $6302 \AA$ lines and came to the conclusion that there is 5 times more horizontal than vertical flux in the quiet photosphere. Subsequently Schüssler \& Vögler (2008) reported that their numerical simulations of magneto-convection also showed a similar preponderance of horizontal flux. In contrast, I have analysed the identical Hinode data set and come to the opposite conclusion, namely that it is the vertical flux that dominates.

The main reason why it is possible to come to such opposite conclusions from the identical data set has to do with the way one deals with the influence of the magnetic structuring on subresolution scales. Due to non-linearities in the relation between polarization and the field properties, the averaging process affects the polarization and the field differently (cf. Stenflo 1994). The non-linearities in the linear polarization are much larger than in the circular polarization. The way in which they affect the spatial averaging over the Hinode 0.3 arcsec resolution element depends critically on the nature of the field structuring on subpixel scales. If this subpixel structuring is dealt with incorrectly (or ignored, as is often the case), large differential scaling errors for the transverse and longitudinal fields will occur, resulting in completely wrong field inclinations. Thus, if the subpixel structuring is neglected, the fields would look much more horizontal than they actually are.

It is beyond the scope of the present paper to go into the quite involved procedure of how to account for the subpixel structuring, but it is based on information extracted from the observed differential Stokes $I$ and $V$ line profile effects in the two Fe I 6301 and $6302 \AA$ lines, modeled with synthetic Stokes spectra representing various realistic, hot and cold atmospheres. Some results of this rather complex analysis are illustrated in Fig. 2 for the angular distribution of field vectors. Since the angular distribution is a steep function of flux density, we have binned the data in terms of apparent flux density in the $6301 \AA$ line. The upper panel of Fig. 2 shows the distribution for the 100-120 G bin. An isotropic distribution of field vectors would be flat in this representation (histogram in terms of $\mu=\cos \gamma$ bins, $\gamma$ being the inclination angle). If horizontal fields would dominate, the distribution would have a negative slope. The steep positive gradient shows that the fields for this flux density bin are strongly peaked around the vertical direction.

To characterize each histogram with a single parameter, we have fitted it with the function $\mu^{\alpha}$, where $\alpha=0$ represents the isotropic case, and the distribution gets increasingly peaked around the vertical direction as $\alpha$ increases. The lower panel in Fig. 2 shows the fitted $\alpha$ values as a function of flux density. For flux densities below $15 \mathrm{G} \alpha$ becomes negative, which would suggest that the weakest flux densities are predominantly horizontal. This is however entirely an artefact of noise, as has been verified by Monte-Carlo simulations using the actual noise values extracted from the Hinode data.

We find from this analysis that the angular distribution is nearly isotropic for the smallest flux densities, but becomes rapidly very peaked around the vertical direction as the flux density increases. It is natural to expect this to happen as a result of the increasing influence of the buoyancy forces.

\section{Probability distributions for the flux densities}

While the subpixel structuring has a profound effect on the extracted transverse flux densities, its influence on the line-of-sight flux densities is much more modest, although it needs to be accounted for. In Fig. 3 we show the histogram or probability density function (PDF) for the vertical flux densities, extracted from the mentioned Hinode data set for the quiet disk center (dashed curve). It can be fitted well by the sum of a symmetric Voigt function and an anti-symmetric line dispersion function, characterized by a "damping 

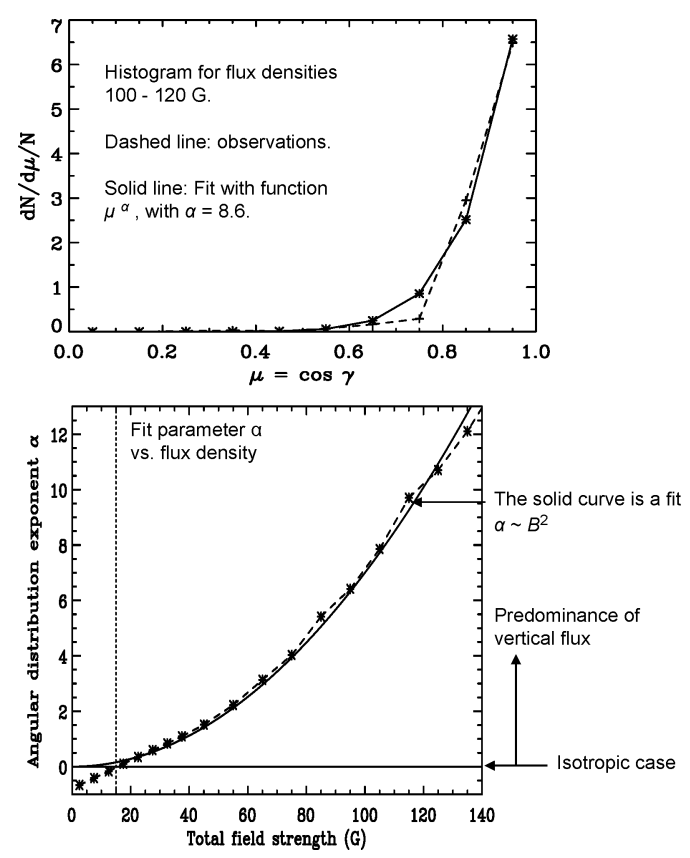

Figure 2. Angular distributions extracted from the Hinode data for the quiet-Sun disk center. Upper panel: Histogram representing the flux density interval 100-120 G. Lower panel: Variation with flux density of fit parameter $\alpha$, showing how the fields get strongly peaked around the vertical direction as the flux density increases. The negative values of $\alpha$ below $15 \mathrm{G}$ can be shown to be an artefact of noise. The Hinode data are therefore consistent with an isotropic distribution for the weakest fluxes.

constant" (to use the terminology from spectral line analysis) and a "Doppler width" characterizing the shape of the inner core. Since the value of the Doppler width closely agrees with its value for a pure Gaussian noise distribution, using the measured value of the noise in the Hinode data, the noise-free PDF is well represented by the mentioned sum of the Voigt and line dispersion functions, if we simply set the Doppler width to zero. The noise-deconvolved PDF (solid curve in Fig. 3) is proportional to the function

$$
\frac{(\gamma / 2)^{2}+0.038 B}{B^{2}+(\gamma / 2)^{2}},
$$

where $B$ is the flux density in $\mathrm{G}$, and $\gamma=8 \mathrm{G}$.

The symmetric (Lorentzian) part of this function is represented by the dotted curve in Fig. 3. The anti-symmetric part accounts for the imbalance between the positive and negative polarities in the solar region that is being sampled.

We note that the PDF has its peak at zero flux density. A non-zero location of the histogram peak that has previously been reported (cf. Lites et al. 2008) is an artefact of the way in which the measurement noise was dealt with. The influence of the noise in the data becomes much more dramatic in the case of the horizontal fields derived from the linear polarization, with the result that the apparent PDFs for the horizontal and total flux densities drop towards zero for the weakest fields. Careful analysis of how the influence of the noise propagates, combined with Monte-Carlo simulations, show however that the apparent, shifted location of the PDF maxima is an artefact of noise. Also in the case of the horizontal and total flux densities, the noise-corrected PDFs all have their maxima at zero flux density. 


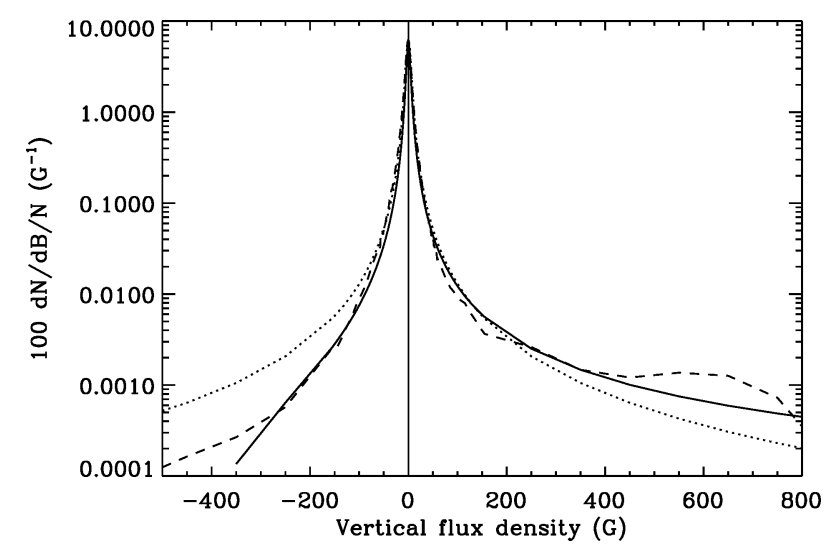

Figure 3. PDF for the vertical flux density, as extracted from the quiet-Sun Hinode data at disk center (dashed curve). The solid curve is a noise-deconvolved analytical representation in terms of a sum of a Lorentz and a line dispersion function, as given by Eq. (4.1). The symmetric part of this function is given by the dotted curve.

\section{Concluding remarks}

It is clear that we need to transcend the 2-component approach in the direction of continuous PDFs. This however requires some modelling assumptions for the behavior of the PDFs in the spatially unresolved domain, where they are not directly observable. To obtain empirical guidance we need to explore the scaling laws of the PDFs in the resolved domain, where we notice a high degree of fractal-like scale invariance, which is likely to continue into the unresolved domain. Even in the resolved domain, however, the polarimetric quantities averaged over the resolution element are strongly influenced by the nature of the subpixel magnetic structuring. Here we have tried to cast some light on these problems and indicate how they may be overcome.

\section{Acknowledgements}

Hinode is a Japanese mission developed and launched by ISAS/JAXA, with NAOJ as domestic partner and NASA and STFC (UK) as international partners. It is operated by these agencies in co-operation with ESA and NSC (Norway).

\section{References}

de Wijn, A. G., Stenflo, J. O., Solanki, S. K., \& Tsuneta, S. 2009, Space Science Reviews, 144, 275 Faurobert-Scholl, M. 1993, A\&A, 268, 765

Lites, B. W., Kubo, M., Socas-Navarro, H., et al. 2008, ApJ, 672, 1237

Schüssler, M. \& Vögler, A. 2008, A\& A, 481, L5

Stenflo, J. O. 1973, Solar Phys. 32, 41

Stenflo, J. O. 1982, Solar Phys. 80, 209

Stenflo, J. O. 1994, Solar Magnetic Fields - Polarized Radiation Diagnostics. (Kluwer)

Stenflo, J. O. 2009, in: K. N. Nagendra, H.-G. Ludwig, \& P. Bonifacio (eds.), 3D views on cool stellar atmospheres: theory meets observation, Proc. JD10 at IAU General Assembly, Rio, Aug. 10-11, 2009, MemSAI, 80, 690

Stenflo, J. O. \& Holzreuter, R. 2002, in: H. Sawaya-Lacoste (ed.), Magnetic Coupling of the Solar Atmosphere, ESA Publ. SP-505, 101

Stenflo, J. O. \& Holzreuter, R. 2003, in: A. A. Pevtsov \& H. Uitenbroek (eds.), Current Theoretical Models and High Resolution Solar Observations, Proc. 21st International NSO/SP Workshop, ASP Conf. Ser. (San Francisco: ASP), vol. 286, 169

Trujillo Bueno, J., Shchukina, N., \& Asensio Ramos, A. 2004, Nature, 430, 326 